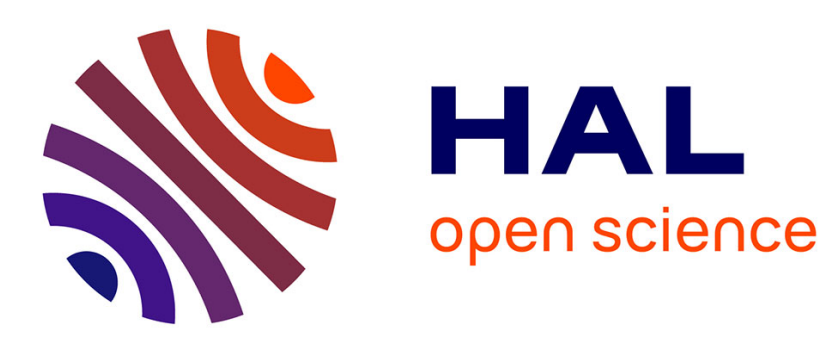

\title{
A theoretical study on cyclacenes: Analytical tight-binding approach
}

\author{
Stefano Battaglia, Hai-anh Le, Gian Luigi Bendazzoli, Noelia Faginas Lago, \\ Thierry Leininger, Stefano Evangelisti
}

\section{- To cite this version:}

Stefano Battaglia, Hai-anh Le, Gian Luigi Bendazzoli, Noelia Faginas Lago, Thierry Leininger, et al. A theoretical study on cyclacenes: Analytical tight-binding approach. International Journal of Quantum Chemistry, 2018, 118 (12), pp.e25569. 10.1002/qua.25569 . hal-01802348

\section{HAL Id: hal-01802348 \\ https://hal.science/hal-01802348}

Submitted on 5 Oct 2018

HAL is a multi-disciplinary open access archive for the deposit and dissemination of scientific research documents, whether they are published or not. The documents may come from teaching and research institutions in France or abroad, or from public or private research centers.
L'archive ouverte pluridisciplinaire HAL, est destinée au dépôt et à la diffusion de documents scientifiques de niveau recherche, publiés ou non, émanant des établissements d'enseignement et de recherche français ou étrangers, des laboratoires publics ou privés. 


\section{ACCEPTED MANUSCRIPT}

This is the peer reviewed version of the following article: Battaglia S, Le H-A, Bendazzoli GL, Faginas-Lago N, Leininger T, Evangelisti S. A theoretical study on cyclacenes: Analytical tight-binding approach. Int J Quantum Chem. 2018;118:e25569. , which has been published in final form at https://doi.org/10.1002/qua.25569. This article may be used for non-commercial purposes in accordance with Wiley Terms and Conditions for Use of Self-Archived Versions.

DOI: https://doi.org/10.1002/qua.25569 


\title{
A Theoretical Study on Cyclacenes: Analytical Tight-Binding Approach
}

\author{
Stefano Battaglia*† Hai-Anh Le $\ddagger$ Gian Luigi Bendazzoli \\ Noelia Faginas-Lago ${ }^{\|}$, Thierry Leininger**'Stefano Evangelisti ${ }^{\dagger \dagger}$
}

September 7, 2018

\begin{abstract}
We present a theoretical study of cyclacene molecules performed at tight-binding level. The orbital energies and eigenvectors have been analytically computed, and exact expressions for the axial component of the total position spread and polarizability tensors have been obtained. In absence of dimerization, the system has a $D_{n h}$ symmetry, where $n$ is the number of hexagonal units. The energy bands present no gap at the Fermi level, and to this fact it corresponds a diverging (per-electron) polarizability for $n \rightarrow \infty$ in the direction of the system symmetry axis. The two (degenerate) components of the polarizability on the $\sigma_{h}$ symmetry plane, on the other hand, remain finite for $n \rightarrow \infty$. The total position spread tensor presents a qualitatively different behavior, since all the three components of the position spread per electron remain finite for $n \rightarrow \infty$. The results are analyzed and discussed for both axial and planar components separately as these are affected differently with respect to the increasing system size. Both dipole polarizability and total position spread have been computed using an $a b$ initio approach for the smallest systems, in order to compare the analytical tight-binding expressions with a higher-level theory.
\end{abstract}

Keywords: Cyclacenes, Tight-Binding, CASSCF, TPS, Polarizability

${ }^{*}$ Laboratoire de Chimie et Physique Quantiques, IRSAMC, Université Paul Sabatier, 118 Route de Narbonne, F-31062 Toulouse Cedex - France

${ }^{\dagger}$ Dipartimento di Chimica, Biologia e Biotecnologie, Università degli Studi di Perugia, Vie Elce di Sotto 8, I-06123 Perugia, Italy

${ }^{\ddagger}$ Department of Chemistry, Northwestern University, 2145 Sheridan Road, Evanston, IL 60208-3113, USA

${ }^{\S}$ Dipartimento di Chimica Industriale "Toso Montanari”, Università di Bologna, Viale Risorgimento 4, I-40136 Bologna, Italy

"Dipartimento di Chimica, Biologia e Biotecnologie, Università degli Studi di Perugia, Vie Elce di Sotto 8, I-06123 Perugia, Italy

"Consortium for Computational Molecular and Materials Sciences (CMS2), Via Elce di Sotto 8, I-06123, Perugia, Italy

${ }^{* *}$ Laboratoire de Chimie et Physique Quantiques, IRSAMC, Université Paul Sabatier, 118 Route de Narbonne, F-31062 Toulouse Cedex - France

${ }^{\dagger \dagger}$ Laboratoire de Chimie et Physique Quantiques, IRSAMC, Université Paul Sabatier, 118 Route de Narbonne, F-31062 Toulouse Cedex - France 


\section{Introduction}

First imagined by Edgar Heilbronner in $1954^{1},[n]$ cyclacenes (cyclic polyacenes) have been a fascinating research topic. A thorough understanding of cyclacenes is important as they could be considered as the shortest zigzag carbon nanotube (CNT) and could be used to model finite-length CNTs in simulations. Furthermore, $[n]$ cyclacene chains have the potential to be used as precursors for the synthesis of zigzag CNTs, allowing for a more controlled way to fabricate this type of carbon nanostructure ${ }^{2}$. Unfortunately, the synthesis of $[n]$ cyclacenes has not yet been realized as it is summarized in a number of reviews ${ }^{3-5}$.

Besides experimental work on $[n]$ cyclacenes, several computational studies using semi-empirical methods ${ }^{6-16}$ and $a b$ initio methods ${ }^{17-22}$ have been carried out on these systems. The semiempirical work was focused both on an analysis of the structural properties of the cyclacenes as well as the behavior of electronic properties such as the HOMO-LUMO gap as a function of the system size parameter $n$. It was found that the gap decreases in an alternating way depending on the parity of $n$, as more units are added to the system. Depending on the type of semi-empirical method employed, different limits for the gap were found, but all in agreement with their general trend. The investigation of geometrical parameters, in particular the $\mathrm{C}-\mathrm{C}$ bond lengths perpendicular as well as parallel to the principal axis and their alternation with respect to different values of $n$, was also insightful. The zigzag bonds form two parallel delocalized polyacetylenic ribbons which are connected through the parallel $\mathrm{C}-\mathrm{C}$ bond. It has been found that the polyacetylenic chains undergo dimerization at AM1 level of theory, while the parallel bond results quite elongated. The influence of such geometric distortion from the non-dimerized system is to uniformly increase the HOMO-LUMO energy gap.

First principles $a b$ initio studies mainly concentrated on the ground-state character of the wave function and the corresponding excitation energy to the first electronically excited state. Recent studies ${ }^{19-22}$ have shown that the ground state is a singlet state of open-shell character, with an increasing number of unpaired electrons with increasing system size. This fact in particular, is believed as one of the causes of the difficulties in the synthesis of cyclacenes as it was already argued in the reviews mentioned above. Another important recent result $^{21,22}$ is the clear behavior of the singlet-triplet energy gap as a function of the system 
size. It was found that for an increasing number $n$ of units, the gap decreases toward a finite value. This property is very similar to their linear counterpart, which shows the same behavior, opening important possible applications also in the field of molecular electronics. The high computational cost of high-level methods based on first principles severely limits the maximal size of the system that can be studied. As a result, semi-empirical approximations as simple as the tight-binding (Hückel) method, remain important to gain insight in the behavior of molecular systems at the thermodynamic limit.

In this article, an analytical investigation of cyclacenes in the Hückel approximation is presented. We go beyond the simple study of the HOMO-LUMO gap by analyzing the behavior of the total position spread (TPS) and polarizability tensors as a function of the system size. The MATHEMATICA software package ${ }^{23}$ was used to obtain the analytical expressions of the density of states, the axial component of the TPS and polarizability tensors as well as the numerical calculation of the corresponding longitudinal components.

Fully analytical expressions for the axial components of the TPS and polarizability tensors were computed. For the planar components, on the other hand, we were not able to obtain closed expressions.

This article is organized as follows. In Section 2, the tight-binding Hamiltonian for a cyclacene system is presented, and its eigenvalues and eigenvectors, along with the density of states, are obtained in closed form. In Section 3, analytical expressions of the axial components of the TPS and polarizability are also derived in closed form. The corresponding planar quantities were computed numerically. The molecular properties thus obtained are then compared to higher level results calculated by ab initio methods. Some conclusions are drawn in Section 4, where the different behavior of the axial polarizability and TPS is discussed.

\section{Cyclic Hückel Polyacenes: the Tight-Binding Approximation}

For two-dimensional (2D) or locally 2D carbon systems, the tight-binding (or Hückel) Hamil-

tonian $\hat{H}$ is a topological operator that involves carbon $\pi$ electrons only. Its parameters are defined as a function of the $\mathrm{C}-\mathrm{C}$ bond distance alone and, in general, the geometry of the 
system has no influence on the eigenvalues and eigenvectors of $\hat{H}$ besides its symmetry. However, in this work we are interested in computing the polarizability and the total position spread tensors, which depend on the fine details of the system geometry. Here, we consider cyclic polyacenes consisting of $n$ benzene hexagons of side length $b$ (thus all $\mathrm{C}-\mathrm{C}$ bonds are

assumed to be equal), lying on a cylinder of radius $R=\frac{n b \sqrt{3}}{2 \pi}$ aligned along the $z$-axis. A schematic drawing of the system can be seen in Figure 1, while the coordinates of the atoms are summarized in Table 1.

\section{Hückel Orbitals and Energies}

We define the unit cell of $[n]$ cyclacenes with four carbon atoms as shown in Figure 2, where orthonormal $p$-like orbitals $p_{\mu}^{\nu}(\mu \in[0, n-1]$ denotes the cell and $\nu \in[1,4]$ the atom within the cell) have been centered on each atom. The orbitals $p_{\mu}^{\nu}$ are assumed to be eigenfunctions of the position operator, thus satisfying

$$
\left\langle p_{\mu}^{\nu}|\hat{r}| p_{\mu^{\prime}}^{\nu^{\prime}}\right\rangle=w_{\mu}^{\nu} \delta_{\nu \nu^{\prime}} \delta_{\mu \mu^{\prime}}
$$

where $r=x, y, z$.

The Hamiltonian is then defined as usual, with the on-site and hopping integrals, $a$ and $t$, respectively, given by

$$
\left\langle p_{\mu}^{\nu}|\hat{H}| p_{\mu}^{\nu}\right\rangle=a
$$

and

$$
\left\langle p_{\mu}^{\nu}|\hat{H}| p_{\mu^{\prime}}^{\nu^{\prime}}\right\rangle=t \delta_{\nu \nu \pm 1} \delta_{\mu \mu \pm 1}
$$

where $\nu^{\prime}=\nu \pm 1$ and $\mu^{\prime}=\mu \pm 1$.

The analytical expression of the four energy bands is known ${ }^{15}$ (see Appendix for a full derivation), given by

$$
\begin{aligned}
& \varepsilon_{1}(\xi)=a-\frac{t}{2}(+1+\sqrt{9+8 \cos \xi}) \\
& \varepsilon_{2}(\xi)=a-\frac{t}{2}(-1+\sqrt{9+8 \cos \xi}) \\
& \varepsilon_{3}(\xi)=a-\frac{t}{2}(+1-\sqrt{9+8 \cos \xi}) \\
& \varepsilon_{4}(\xi)=a-\frac{t}{2}(-1-\sqrt{9+8 \cos \xi})
\end{aligned}
$$


where $\xi=k \theta, k$ is an integer number and $\theta=2 \pi / n$.

Note that since $-t<0$, the eigenvalues are ordered from the smallest $\left(\varepsilon_{1}\right)$ to the largest $\left(\varepsilon_{4}\right)$, for any value of $k$. The eigenvectors of the full Hamiltonian for an arbitrary finite $[n]$ cyclacene are also analytically known, and given by

$$
|m, k\rangle=\frac{1}{\sqrt{n}} \sum_{\mu=0}^{n-1} e^{\frac{2 \pi i k \mu}{n}}\left[c_{1, m}(k \theta) p_{\mu}^{1}+c_{2, m}(k \theta) p_{\mu}^{2}+c_{3, m}(k \theta) p_{\mu}^{3}+c_{4, m}(k \theta) p_{\mu}^{4}\right]
$$

where $m$ and $k$ are quantum numbers related to the symmetry of the system. Note that the eigenvectors with $m=1,3$ are symmetric with respect to the $\sigma_{h}$ plane, while antisymmetric for $m=2,4$ (see Appendix for details).

By plotting the four energy eigenvalues given in Equation (4) as functions of $\xi$ between $-\pi$ and $\pi$, we get the energy bands depicted in Figure 3 for $a=0$ and $t=1$.

The difference between the lowest unoccupied and highest occupied band is given by

$$
\Delta(\xi)=\varepsilon_{3}(\xi)-\varepsilon_{2}(\xi)=t(\sqrt{9+8 \cos (\xi)}-1)
$$

where we notice the closure of the gap for $\xi=\pi$. The two eigenvalues $\varepsilon_{2}(\pi)$ and $\varepsilon_{3}(\pi)$ are degenerate at this level of theory.

In finite systems, the size of the gap depends on the integer value $k$ : the gap vanishes if and only if there exists a $k$ such that $\xi=k \theta=\pi$. Since $\theta=2 \pi / n$, this implies that $n$ must be even.

To obtain the partial density of states (PDOS) for each energy band, it is convenient to consider $n \rightarrow \infty$ and to treat $\xi$ as a continuous variable defined as the inverse function of $\varepsilon_{m}(\xi)$. The PDOS is then obtained for each band separately by computing the derivative as exemplified here for the highest occupied band

$$
\frac{\partial \xi}{\partial \varepsilon_{2}}=\frac{\partial}{\partial \varepsilon_{2}} \arccos \left[\frac{1}{2}\left(\frac{\varepsilon_{2}}{t}-\frac{a}{t}-\frac{1}{2}\right)^{2}-\frac{9}{8}\right]=\frac{-\left(\frac{\varepsilon_{2}}{t}-\frac{a}{t}-\frac{1}{2}\right)}{t \sqrt{1-\left[\frac{1}{2}\left(\frac{\varepsilon_{2}}{t}-\frac{a}{t}-\frac{1}{2}\right)^{2}-\frac{9}{8}\right]^{2}}}
$$

The expressions for the other bands are available in the Appendix.

The total density of states of both the occupied bands $\varepsilon_{1}$ and $\varepsilon_{2}$ and the unoccupied bands $\varepsilon_{3}$ and $\varepsilon_{4}$ is reported in Figure 4 for $a=0$ and $t=1$. 
The total energy for a finite-size system composed of $n$ elementary cells is given by the sum of the energies of the occupied orbitals

$$
\mathcal{E}_{\text {tot }}=2 \sum_{k=0}^{n-1}\left[\varepsilon_{1}(k \theta)+\varepsilon_{2}(k \theta)\right]=2 \sum_{k=0}^{n-1}[2 \alpha-t \sqrt{9+8 \cos k \theta}]
$$

where the factor 2 in front of the sum arises from the fact that at every point $k \theta$ on the energy bands there are two electrons. The discrete expression for the energy in Equation (8) can be evaluated in closed form for $n \rightarrow \infty$ as

$$
\mathcal{E}_{\text {tot }}=\frac{n}{\pi} \int_{-\pi}^{\pi}\left[\varepsilon_{1}(\xi)+\varepsilon_{2}(\xi)\right] d \xi=4 n\left(\alpha-\frac{\sqrt{17} t E\left[\frac{16}{17}\right]}{\pi}\right)
$$

The function $E[x]$ is the complete elliptic integral of $2^{\text {nd }}$ kind.

\section{The Total Position Spread and Polarizability Tensors}

The total position spread (TPS) tensor is a key quantity in the theory of polarizability. It was introduced by Resta and co-workers under the name of localization tensor (LT) ${ }^{24-26}$ (notice that Resta's definition differs from ours in a factor equal to the number of electrons). In molecular systems, the TPS tensor gives interesting information on the nature of a bond $^{27,28}$. On the other hand, at thermodynamic limit, the LT (i.e. the TPS divided by the number of electrons) differentiate insulators from conductors according to its asymptotic behavior with respect to the system size.

The TPS tensor is defined as the second moment cumulant of the total position operator

$$
\Lambda_{r r^{\prime}}=\left\langle\Phi_{0}\left|\hat{R}_{r} \hat{R}_{r^{\prime}}\right| \Phi_{0}\right\rangle-\left\langle\Phi_{0}\left|\hat{R}_{r}\right| \Phi_{0}\right\rangle\left\langle\Phi_{0}\left|\hat{R}_{r^{\prime}}\right| \Phi_{0}\right\rangle
$$

where $\hat{R}_{r}=\sum_{i=1}^{N} \hat{r}(i)$ is the sum over the one-electron position operators (with $r, r^{\prime}=x, y$ and $z), N$ the number of electrons and $\left|\Phi_{0}\right\rangle \equiv|0\rangle$ the state of interest (usually the ground state).

The TPS tensor can be conveniently expressed as sum over excited states $\left|\Phi_{I}\right\rangle \equiv|I\rangle$ of the many electron system

$$
\Lambda_{r r^{\prime}}=\sum_{I}\left\langle 0\left|\hat{R}_{r}\right| I\right\rangle\left\langle I\left|\hat{R}_{r^{\prime}}\right| 0\right\rangle-\left\langle 0\left|\hat{R}_{r}\right| 0\right\rangle\left\langle 0\left|\hat{R}_{r^{\prime}}\right| 0\right\rangle
$$


which in the case of the diagonal components $\left(r=r^{\prime}\right)$, simplifies to

$$
\Lambda_{r r}=\sum_{I>0}\left|\left\langle 0\left|\hat{R}_{r}\right| I\right\rangle\right|^{2}
$$

where we note that the second term on the right-hand side of Equation (10) drops out. One advantage of expressing the TPS in the form of Equation (12) is that the polarizability

can be easily obtained by dividing the term $\left|\left\langle 0\left|\hat{R}_{r}\right| I\right\rangle\right|^{2}$ in the sum by the energy difference between the excited state $|I\rangle$ and the reference state $|0\rangle$, resulting in

$$
\alpha_{r r}=\sum_{I>0} \frac{\left|\left\langle 0\left|\hat{R}_{r}\right| I\right\rangle\right|^{2}}{\mathcal{E}_{I}-\mathcal{E}_{0}}
$$

This approach is particularly useful in the case of a model Hamiltonian, since the whole set of many-particle states $|I\rangle$ is known and spans the complete space. In other words, the use of this technique gives the exact value of the TPS and the polarizability.

For a Hückel system and a one-electron operator $\hat{r}$ the only non-zero matrix elements appearing in Equations (12) and (13) are those between the ground state $|0\rangle$ and single excitations thereof. Therefore they reduce to integrals between occupied and empty orbitals. In the case of cyclacenes as modeled in this work, the total position spread tensor takes the following form

$$
\sum_{I>0}\left|\left\langle 0\left|\hat{R}_{r}\right| I\right\rangle\right|^{2}=2 \sum_{m=1}^{2} \sum_{k=0}^{n-1} \sum_{m^{\prime}=3}^{4} \sum_{k^{\prime}=0}^{n-1}\left|\left\langle m, k|\hat{r}| m^{\prime}, k^{\prime}\right\rangle\right|^{2}
$$

where non-primed indexes run over occupied states, primed ones over empty states and the factor 2 in front comes from the double electron occupancy of the orbitals.

\section{The Axial Component of the TPS and the Polarizability}

As usual in Hückel treatments, we assume that Equation (1) holds, such that the matrix representation of $\hat{z}$ in the elementary cell is given by

$$
\mathbf{z}=b\left[\begin{array}{cccc}
1 & 0 & 0 & 0 \\
0 & \frac{1}{2} & 0 & 0 \\
0 & 0 & -\frac{1}{2} & 0 \\
0 & 0 & 0 & -1
\end{array}\right]
$$


The matrix elements appearing in Equation (14) for $\hat{r}=\hat{z}$ do not vanish only for $k=k^{\prime}$ and when the symmetry with respect to $\sigma_{h}$ of the orbitals in the sum is different. Thus, the only nonzero integrals in this case are

$$
\begin{aligned}
& \langle 1, k|\hat{z}| 4, k\rangle=\frac{b}{4}\left(-1+\frac{3}{\sqrt{9+8 \cos \xi}}\right) \\
& \langle 2, k|\hat{z}| 3, k\rangle=\frac{b}{4}\left(-1-\frac{3}{\sqrt{9+8 \cos \xi}}\right)
\end{aligned}
$$

and are reported as a function of $\xi$ in Figure 5 (with $b=1$ ), together with their squared value (which according to Equation (14) is the actual contribution entering the defnition of the TPS and the polarizability).

The fact that the integral contributions to $\Lambda_{z z}$ are finite at every point $\xi$ comprised in the interval $(-\pi, \pi]$ already suggests that the behavior of the position spread, as the size of the system increases, will be linear.

The total axial component of the TPS, obtained by summing the integrals in Equations (16) and (17) for all values of $k$, has the following form

$$
\begin{aligned}
\Lambda_{z z} & =2 \sum_{k=0}^{n-1}\left[|\langle 1, k|\hat{z}| 4, k\rangle|^{2}+|\langle 2, k|\hat{z}| 3, k\rangle|^{2}\right] \\
& =\frac{b^{2}}{4} \sum_{k=0}^{n-1}\left(1+\frac{9}{9+8 \cos k \theta}\right)
\end{aligned}
$$

By taking the limit of large $n$ values, Equation (18) can be given in closed form as

$$
\Lambda_{z z}=\frac{n}{2 \pi} \int_{-\pi}^{\pi} 2\left[|\langle 1, k|\hat{z}| 4, k\rangle|^{2}+|\langle 2, k|\hat{z}| 3, k\rangle|^{2}\right] d \xi=\frac{n b^{2}}{68}(17+9 \sqrt{17})
$$

Indeed, $\Lambda_{z z}$ is linear in $n$ and diverges in the thermodynamic limit: $\Lambda_{z z} \rightarrow \infty$ for $n \rightarrow \infty$. The connection to the LT tensor in the theory of the insulating state follows directly, since simply dividing $\Lambda_{z z}$ by the number of electrons in the system (i.e. $4 n$ ) gives a finite number. Accordingly, this means that along the $z$ Cartesian coordinate, the cyclacene can be regarded as an insulator. This can be understood as the electrons fluctuation along the $z$ direction remaining bounded while the system is growing in the other two Cartesian coordinates as $n$ increases.

To compute the $z z$ component of the polarizability, the transition matrix elements $\langle 1, k|\hat{z}| 4, k\rangle$ 
and $\langle 2, k|\hat{z}| 3, k\rangle$ have to be divided by $\varepsilon_{4}-\varepsilon_{1}$ and $\varepsilon_{3}-\varepsilon_{2}$, respectively. This results in

$$
\frac{|\langle 1, k|\hat{z}| 4, k\rangle|^{2}}{\varepsilon_{4}-\varepsilon_{1}}=\frac{b^{2}\left(-1-\frac{3}{\sqrt{9+8 \cos \xi}}\right)^{2}}{16 t(1+\sqrt{9+8 \cos \xi})}
$$

and

$$
\frac{|\langle 2, k|\hat{z}| 3, k\rangle|^{2}}{\varepsilon_{3}-\varepsilon_{2}}=\frac{b^{2}\left(-1-\frac{3}{\sqrt{9+8 \cos \xi}}\right)^{2}}{16 t(-1+\sqrt{9+8 \cos \xi})}
$$

The polarizability contributions of Equations (20) and (21) are shown in Figure 6 for $b=1$ and $t=1$.

Differently to the TPS case, the $2 \rightarrow 3$ transition element diverges at $\xi=\pi$ due to the vanishing gap in the denominator. This can also be seen from a power series expansion around that point, reading

$$
\frac{|\langle 2, k|\hat{z}| 3, k\rangle|^{2}}{\varepsilon_{3}-\varepsilon_{2}}=\frac{b^{2}}{t}\left[\frac{1}{2(\xi-\pi)^{2}}-\frac{23}{24}+\frac{1741(\xi-\pi)^{2}}{480}-\frac{168461(\xi-\pi)^{4}}{12096}\right]+O\left[(\xi-\pi)^{5}\right]
$$

The direct consequence of the diverging contribution is that not only the total $z z$ component of the polarizability tensor trivially diverges in thermodynamic limit (similarly to the TPS)

$$
\alpha_{z z}=\frac{n}{2 \pi} \int_{-\pi}^{\pi} 2\left[\frac{|\langle 2, k|\hat{z}| 3, k\rangle|^{2}}{\varepsilon_{3}-\varepsilon_{2}}+\frac{|\langle 1, k|\hat{z}| 4, k\rangle|^{2}}{\varepsilon_{4}-\varepsilon_{1}}\right] d \xi \rightarrow \infty
$$

but also the per-electron polarizability diverges

$$
\frac{\alpha_{z z}}{4 n}=\frac{1}{8 \pi} \int_{-\pi}^{\pi} 2\left[\frac{|\langle 2, k|\hat{z}| 3, k\rangle|^{2}}{\varepsilon_{3}-\varepsilon_{2}}+\frac{|\langle 1, k|\hat{z}| 4, k\rangle|^{2}}{\varepsilon_{4}-\varepsilon_{1}}\right] d \xi \rightarrow \infty
$$

since the first of the two integrands does so too (cf. Equation (22)).

This means that $\alpha_{z z}$ does not simply diverge (linearly) because of the $n / 2 \pi$ prefactor in Equation (23), but faster than that, as the integral itself is unbounded. This result appears in contradiction with the axial component of the per-electron TPS, which approaches a finite value as $n \rightarrow \infty$.

We argue that the discrepancy of the two quantities is due to the limitation of the tightbinding approximation.

In order to investigate this incongruence, as well as obtaining more quantitative results for both the TPS and polarizability, complete active space self-consistent field (CASSCF) calculations have been performed on a limited number of $[n]$ cyclacenes (geometries and active 
spaces taken from Ref. 22) using the $\mathrm{MOLPRO}^{29,30}$ and $\mathrm{ORCA}^{31}$ program packages.

In Figure 7, the axial component of the TPS (as implemented in MOLPRO ${ }^{27,32}$ ) computed using a double- $\zeta$ atomic natural orbital basis set with polarization functions (ANO-DZP) ${ }^{33}$ is depicted as function of the system size. Although the predicted linear behavior of $\Lambda_{z z}$ is an asymptotic limit, already for the first (even-numbered) $[n]$ cyclacenes, a clear linear trend is observed with a fully quantum mechanical approach, thus supporting the tight-binding results.

The polarizability has somewhat higher computational requirements. In this work we have computed the polarizability tensor only for cyclacenes with $n$ equal $6,8,10$ and 12 , using the aug-cc-pVDZ basis set and the resolution-of-the-identity approximation for the molecular orbital transformation ${ }^{34-36}$. The threshold applied for convergence was set to "verytight" and an electric field perturbation of 0.0001 atomic units has been added to the system in order to obtain the polarizability by finite differentiation of the dipole moment.

The results for the $\alpha_{z z}$ component are listed in Table 2. The four available ab initio polarizability values appear to increase approximately linearly with system size. In particular, there is no sign suggesting the divergence observed at tight-binding level. This is not surprising, as the problematic term in Equation (24) is due to the vanishing denominator of the $2 \rightarrow 3$ transition integral.

Already Hartree-Fock theory predicts a non-degenerate HOMO-LUMO pair, suggesting, remaining within an uncoupled Hartree-Fock picture to obtain the static polarizability, that the problematic denominator responsible for divergence in Equation (24) would have a finite value and thus not cause any trouble.

A similar argument is probably less accurate at CASSCF level, where the monodeterminantal picture is lost and orbital energies are not well-defined anymore. Nevertheless, natural orbital occupation numbers (NOONs) of the HOMO and LUMO (last column of Table 2) differ significantly for the cyclacenes considered here, suggesting a clear difference between them, unlike in the case of the degenerate Hückel orbitals. To strictly apply the sum-overstates formula given in Equation (13) to the CASSCF formalism, one would have to compute all excited states, which is clearly unfeasible (and likely very inaccurate).

The above arguments however, provide some evidence that the missing electron-electron 
repulsion within the tight-binding approximation is responsible for the degeneracy at the Fermi level, ultimately producing the unexpected divergence of Equation (24).

\section{The Planar Components of the TPS and the Polarizability}

To evaluate the planar components of the TPS and polarizability, instead of working with the two components $\hat{x}$ and $\hat{y}$ separately, it is more convenient to consider the combined operator $\hat{x} \pm i \hat{y}$ in its polar form given by $\hat{R} e^{ \pm i \hat{\varphi}}$. In this notation, $\varphi$ is the angle of rotation about the $z$ axis and $R$ the radius of the cyclacene. The angle $\varphi$ formally depends on the elementary cell $\mu$ and is defined as

$$
\varphi= \begin{cases}\frac{2 \pi \mu}{n} & \text { if } \nu=2,3 \\ \frac{2 \pi\left(\mu+\frac{1}{2}\right)}{n} & \text { if } \nu=1,4\end{cases}
$$

such that, when Equation (1) holds, the matrix form of the operator $\hat{x} \pm i \hat{y}$ expressed in the reduced basis spanned by $p_{\mu}^{\nu}$ is given by

$$
\mathbf{x} \pm i \mathbf{y}=R e^{ \pm \frac{i 2 \pi \mu}{n}}\left[\begin{array}{cccc}
e^{ \pm \frac{i \pi}{n}} & 0 & 0 & 0 \\
0 & 1 & 0 & 0 \\
0 & 0 & 1 & 0 \\
0 & 0 & 0 & e^{ \pm \frac{i \pi}{n}}
\end{array}\right]
$$

and $R$ remains a simple multiplicative constant as defined at the beginning of this article, i.e. $R=\frac{n b \sqrt{3}}{2 \pi}$. The application of this operator on the many-particle state $|m, k\rangle$ results in

$$
R e^{ \pm i \hat{\varphi}}|m, k\rangle=\frac{R}{\sqrt{n}} \sum_{\mu=0}^{n-1} e^{\frac{2 i \pi \mu}{n}(k \pm 1)}\left[e^{ \pm \frac{i \pi}{n}} c_{1, m} p_{\mu}^{1}+c_{2, m} p_{\mu}^{2}+c_{3, m} p_{\mu}^{3}+e^{ \pm \frac{i \pi}{n}} c_{4, m} p_{\mu}^{4}\right]
$$

This implies that the non-vanishing matrix elements are given by

$$
\left\langle m, k|\hat{x}+i \hat{y}| m^{\prime}, k-1\right\rangle \quad \text { and } \quad\left\langle m, k|\hat{x}-i \hat{y}| m^{\prime}, k+1\right\rangle
$$

where the factor $e^{ \pm \frac{i 2 \pi \mu}{n}}$ in Equation (26) allows interactions only between states with $k^{\prime}-k=$ \pm 1 . Moreover, differently to the case of the $\hat{z}$ operator, now $|m, k\rangle$ and $\left|m^{\prime}, k^{\prime}\right\rangle$ must be of the same symmetry with respect to $\sigma_{h}$ for the integrals to be nonzero. Thus, the contributing 
transitions to the planar components of the TPS are $1 \rightarrow 3$ and $2 \rightarrow 4$.

This makes a total of four types of integrals different from zero, which correspond to

$$
\begin{array}{rrr}
\langle 1, k|\hat{x}+i \hat{y}| 3, k-1\rangle & \text { and } \quad\langle 1, k|\hat{x}-i \hat{y}| 3, k+1\rangle \\
\left\langle 2, k|\hat{x}+i \hat{y}| 4^{\prime}, k-1\right\rangle & \text { and } \quad\left\langle 2, k|\hat{x}-i \hat{y}| 4^{\prime}, k+1\right\rangle
\end{array}
$$

where we point out that these elements posses an analytical expression, although quite cumbersome. For this reason they are omitted here and given in the Appendix.

The rotational symmetry of the cyclacene simplifies the calculation of the individual integrals of $\hat{x}$ and $\hat{y}$, since these are simply given by

$$
\begin{aligned}
\left\langle m, k|\hat{x}| m^{\prime}, k-1\right\rangle & =\left\langle m, k|\hat{y}| m^{\prime}, k-1\right\rangle=\frac{1}{2}\left\langle m, k|\hat{x}+i \hat{y}| m^{\prime}, k-1\right\rangle \\
\left\langle m, k|\hat{x}| m^{\prime}, k+1\right\rangle & =\left\langle m, k|\hat{y}| m^{\prime}, k+1\right\rangle=\frac{1}{2}\left\langle m, k|\hat{x}-i \hat{y}| m^{\prime}, k+1\right\rangle
\end{aligned}
$$

Moreover, the squared integrals contributing to the planar component of the TPS are the same for both transitions $1 \rightarrow 3$ and $2 \rightarrow 4$ and are shown in Figure 8 as exemplified by the term $\frac{1}{4} \cdot\left|\left\langle 2, k|\hat{x}+i \hat{y}| 4^{\prime}, k-1\right\rangle\right|^{2}=\left|\left\langle 2, k|\hat{x}| 4^{\prime}, k-1\right\rangle\right|^{2}=\left|\left\langle 2, k|\hat{y}| 4^{\prime}, k-1\right\rangle\right|^{2}$.

Furthermore, the rotational symmetry implies that the two planar components of the TPS tensor are degenerate, given by

$$
\begin{aligned}
\Lambda_{x x}=\Lambda_{y y}=\frac{1}{2} \sum_{k=0}^{n-1} & {\left[|\langle 2, k|\hat{x}+i \hat{y}| 4, k-1\rangle|^{2}+|\langle 2, k|\hat{x}-i \hat{y}| 4, k+1\rangle|^{2}\right.} \\
& \left.+|\langle 1, k|\hat{x}+i \hat{y}| 3, k-1\rangle|^{2}+|\langle 1, k|\hat{x}-i \hat{y}| 3, k+1\rangle|^{2}\right]
\end{aligned}
$$

With the same approach applied to the axial component, the polarizabilities $\alpha_{x x}$ and $\alpha_{y y}$ are obtained by dividing the matrix elements by the energy differences

$$
\begin{aligned}
\alpha_{x x}=\alpha_{y y}=\frac{1}{2} \sum_{k=0}^{n-1} & {\left[\frac{|\langle 2, k|\hat{x}+i \hat{y}| 4, k-1\rangle|^{2}}{\varepsilon_{4}(k \theta-\theta)-\varepsilon_{2}(k \theta)}+\frac{|\langle 2, k|\hat{x}-i \hat{y}| 4, k+1\rangle|^{2}}{\varepsilon_{4}(k \theta+\theta)-\varepsilon_{2}(k \theta)}\right.} \\
+ & \left.\frac{|\langle 1, k|\hat{x}+i \hat{y}| 3, k-1\rangle|^{2}}{\varepsilon_{3}(k \theta-\theta)-\varepsilon_{1}(k \theta)}+\frac{|\langle 1, k|\hat{x}-i \hat{y}| 3, k+1\rangle|^{2}}{\varepsilon_{3}(k \theta+\theta)-\varepsilon_{1}(k \theta)}\right]
\end{aligned}
$$

where in this case the dependence of the denominator on $k \theta$ is crucial and therefore explicitly written.

We were not able to express the two previous equations in closed form as we have done for the axial component. For this reason we resort on numerical computation to inspect the 
behavior at the thermodynamic limit.

The results for the spread and the polarizability are reported in Figure 9, where we show, in logarithmic scale, the results of a numerical evaluation of both quantities up to a few thousands of monomer units. It appears clear that the planar components of both the perelectron total position spread (i.e. the localization tensor) and the per-electron polarizability tend to a finite value as the number of monomer units grows to infinity. The limit is reached quite quickly, and it does not change significantly beyond the system size considered.

In Table 2 the values for $\alpha_{x x}$ computed at CASSCF/aug-cc-pVDZ level are listed. The behavior for the first few points of the polarizability appears linear, but clearly the limited number of systems analyzed are not enough to infer a trend.

\section{Conclusions}

The analytical expression for the eigenvalues and eigenvectors of cyclacenes in the tightbinding approximation has been obtained. By using the MATHEMATICA software package, the exact expression of the energy bands, density of states, axial position spread and polarizability have been computed. The energy gap vanishes at the Fermi level, as it is located at the frontier between two different bands. For this reason, the density of states shows the presence of a Van Hove singularity at the Fermi level.

The axial per-electron TPS remains finite, in accordance with the finite extension of the system in this direction. Quite remarkably, however, the corresponding per-electron polarizability diverges, a behavior that is commonly found only in the case of conductors. In general a divergence of the per-electron polarizability is associated to a similar behavior of the corresponding position spread tensor. To our knowledge, this is the only case where the two quantities do not behave in the same way. We argue that the reason is likely due to the missing description of the electron-electron repulsion in the tight-binding approximation, which removes (at ab initio level of theory) the degeneracy present at the Fermi level. This is supported by CASSCF calculations of the dipole polarizability for a few small systems.

The axial component of the TPS has been computed at CASSCF level of theory as well, and remarkably, for the series of $[n]$ cyclacenes from $n=6$ to $n=22$, it increases linearly with 
system size as predicted by the analytical expression derived within Hückel theory, despite being an asymptotic limit.

We were not able to obtain analytically exact expression for the planar (degenerate) components of the spread and polarizability tensors. A numerical calculation, however, does not show any evidence of a divergence of these quantities. Therefore the system is predicted to be, at this level of description, an insulator. This result is of particular interest, as this material has a zero gap along with a nonzero density of states at the Fermi level, thus suggesting a metallic behavior. The here computed properties however, which depend on the geometrical details of the system, would characterize it as insulator, meaning that closing a linear polyacene to form a ring appears to change the type of the material from metallic to non-metallic, although more investigation is necessary.

In a recent paper ${ }^{22}$, we have extensively studied $[n]$ cyclacenes at an ab initio level. In particular, due to the presence of quasi-degenerate orbitals at the Fermi level, a CASSCF approach is highly recommended. Because of the size of the system, the treatment of all the valence $\pi$ orbitals as active ones is out of question is one would like to study systems beyond $n \approx 16$, even with modern approaches such as the density matrix renormalization group. The knowledge of the structure of the $\pi$ bands obtained in this work is extremely useful in order to perform a rational choice of the active space at the $a b$ initio level.

\section{Acknowledgements}

This work has received funding from the European Union's Horizon 2020 research and innovation programme under the Marie Skłodowska-Curie grant agreement $\mathrm{n}^{\circ} 642294$. We also acknowledge the "Programme Investissements d'Avenir" ANR-11- IDEX-0002-02, reference ANR-10-LABX-0037-NEXT for financial support. H.-A. L. acknowledges the support of the "Theoretical Chemistry and Computational Modelling" (TCCM) Erasmus-Plus Master program. N. F.-L. acknowledges financial support from Fondazione Cassa di Risparmio di Perugia (P 2014/1255, ACT 2014/6167). The calculations of this work have been partly performed by using the resources of the HPC center CALMIP, under the grant 2016-p1048. 


\section{References}

1. E. Heilbronner, Helv. Chim. Acta 37, 921 (1954), ISSN 15222675.

2. H. Omachi, Y. Segawa, and K. Itami, Acc. Chem. Res. 45, 1378 (2012), ISSN 00014842.

3. K. Tahara and Y. Tobe, Chem. Rev. 106, 5274 (2006), ISSN 00092665.

4. R. Gleiter, B. Esser, and S. C. Kornmayer, Acc. Chem. Res. 42, 1108 (2009), ISSN 00014842 .

5. D. Eisenberg, R. Shenhar, and M. Rabinovitz, Chem. Soc. Rev. 39, 2879 (2010), ISSN $1460-4744$.

6. I. Gutman, Polycycl. Aromat. Compd. 8, 251 (1996), ISSN 1040-6638.

7. I. Gutman, P. U. Biedermann, V. Ivanov-Petrović, and I. Agranat, Polycycl. Aromat. Compd. 8, 189 (1996), ISSN 1040-6638.

8. L. Türker, Polycycl. Aromat. Compd. 8, 67 (1996), ISSN 10406638.

9. L. Türker, J. Mol. Struct. 407, 217 (1997).

10. L. Türker, Turkish J. Chem. 22, 109 (1998), ISSN 13000527.

11. L. Türker, J. Mol. Struct. THEOCHEM 454, 83 (1998), ISSN 01661280.

12. L. Türker, J. Mol. Struct. THEOCHEM 491, 275 (1999), ISSN 01661280.

13. L. Türker, J. Mol. Struct. THEOCHEM 531, 333 (2000), ISSN 01661280.

14. L. Türker and S. Gümüs, J. Mol. Struct. THEOCHEM 685, 1 (2004), ISSN 01661280.

15. J.-M. André, B. Champagne, E. A. Perpète, and M. Guillaume, Int. J. Quantum Chem. 84, $607(2001)$.

16. M. Guillaume, B. Champagne, E. A. Perpète, and J.-M. André, Theor. Chem. Acc. 105, 431 (2001), ISSN 1432881X. 
17. H. S. Choi and K. S. Kim, Angew. Chemie - Int. Ed. 38, 2256 (1999), ISSN 14337851.

18. K. N. Houk, P. S. Lee, and M. Nendel, J. Org. Chem. 66, 5517 (2001), ISSN 00223263.

19. Z. Chen, D. E. Jiang, X. Lu, H. F. Bettinger, S. Dai, P. v. R. Schleyer, and K. N. Houk, Org. Lett. 9, 5449 (2007), ISSN 15237060.

20. D. Sadowsky, K. McNeill, and C. J. Cramer, Faraday Discuss. 145, 507 (2010), ISSN 1359-6640.

21. C.-S. Wu, P.-Y. Lee, and J.-D. Chai, Nat. Sci. Reports 6, 37249 (2016), ISSN 20452322.

22. S. Battaglia, N. Faginas-Lago, D. Andrae, S. Evangelisti, and T. Leininger, J. Phys. Chem. A 121, 3746 (2017), ISSN 1089-5639.

23. I. Wolfram Research, Mathematica (2017).

24. R. Resta and S. Sorella, Phys. Rev. Lett. 82, 370 (1999), ISSN 0031-9007.

25. R. Resta, J. Chem. Phys. 124, 104104 (2006), ISSN 0021-9606.

26. R. Resta, Eur. Phys. J. B 79, 121 (2011), ISSN 1434-6028.

27. O. Brea, M. El Khatib, C. Angeli, G. L. Bendazzoli, S. Evangelisti, and T. Leininger, J. Chem. Theory Comput. 9, 5286 (2013), ISSN 15499618.

28. O. Brea, M. El Khatib, G. L. Bendazzoli, S. Evangelisti, T. Leininger, and C. Angeli, J. Phys. Chem. A 120, 5230 (2016), ISSN 1089-5639.

29. H.-J. Werner, P. J. Knowles, G. Knizia, F. R. Manby, M. Schütz, P. Celani, W. Győrffy, D. Kats, T. Korona, R. Lindh, et al., MOLPRO, version 2015.1, a package of ab initio programs (2015), URL http://www.molpro.net.

30. H.-J. Werner, P. J. Knowles, G. Knizia, F. R. Manby, and M. Schütz, Wiley Interdiscip. Rev. Comput. Mol. Sci. 2, 242 (2012), ISSN 17590876.

31. F. Neese, Wiley Interdiscip. Rev. Comput. Mol. Sci. p. e1327 (2017), ISSN 17590876. 
32. M. El Khatib, T. Leininger, G. L. Bendazzoli, and S. Evangelisti, Chem. Phys. Lett. 591, 58 (2014), ISSN 00092614.

33. P.-O. Widmark, P.-A. Malmqvist, and B. O. Roos, Theor. Chim. Acta 77, 291 (1990), ISSN 0040-5744.

34. T. H. Dunning Jr., J. Chem. Phys. 90, 1007 (1989), ISSN 00219606.

35. R. A. Kendall, T. H. Dunning, and R. J. Harrison, J. Chem. Phys. 96, 6796 (1992).

36. F. Weigend, A. Köhn, and C. Hättig, J. Chem. Phys. 116, 3175 (2002), ISSN 0021-9606. 


\section{A The Tight-Binding Hamiltonian}

The mono-electronic tight-binding Hamiltonian is basically parametrized according to the $\mathrm{C}-\mathrm{C}$ bond length, which in this work is assumed to be the same for any $\mathrm{C}-\mathrm{C}$ bond of the cyclacenes. Moreover, the symmetries of these molecules are also retained by the Hamiltonian, and here, we exploit $i$ ) the rotation $C_{n}$ around the $z$-axis and $i i$ ) the reflection plane $\sigma_{h}$ perpendicular to the $z$-axis (plane of the ring).

The Hamiltonian matrix $\mathbf{H}_{n}$ of a cyclic polyacene containing $n$ hexagonal units, expressed in the orthonormal basis of the $p$-like orbitals, is then given by a block circulant matrix of dimension $4 n \times 4 n$ partitioned in $n^{2}$ blocks of size $4 \times 4$

$$
\mathbf{H}_{n}=\left[\begin{array}{cccccc}
\mathbf{H}_{0} & \mathbf{H}_{1} & \mathbf{0} & \ldots & \mathbf{0} & \mathbf{H}_{-1} \\
\mathbf{H}_{-1} & \mathbf{H}_{0} & \mathbf{H}_{1} & \ddots & \vdots & \mathbf{0} \\
\mathbf{0} & \mathbf{H}_{-1} & \ddots & \ddots & \mathbf{0} & \vdots \\
\vdots & \mathbf{0} & \ddots & \ddots & \mathbf{H}_{1} & \mathbf{0} \\
\mathbf{0} & \vdots & \ddots & \mathbf{H}_{-1} & \mathbf{H}_{0} & \mathbf{H}_{1} \\
\mathbf{H}_{1} & \mathbf{0} & \ldots & \mathbf{0} & \mathbf{H}_{-1} & \mathbf{H}_{0}
\end{array}\right]
$$

The structure of the block $\mathbf{H}_{0}$ on the diagonal is

$$
\mathbf{H}_{0}=\left[\begin{array}{cccc}
a & -t & 0 & 0 \\
-t & a & -t & 0 \\
0 & -t & a & -t \\
0 & 0 & -t & a
\end{array}\right]
$$

while the blocks $\mathbf{H}_{1}$ and $\mathbf{H}_{-1}$ are given by

$$
\mathbf{H}_{1}=\left[\begin{array}{cccc}
0 & -t & 0 & 0 \\
0 & 0 & 0 & 0 \\
0 & 0 & 0 & 0 \\
0 & 0 & -t & 0
\end{array}\right] \text { and } \mathbf{H}_{-1}=\left[\begin{array}{cccc}
0 & 0 & 0 & 0 \\
-t & 0 & 0 & 0 \\
0 & 0 & 0 & -t \\
0 & 0 & 0 & 0
\end{array}\right]=\left(\mathbf{H}_{1}\right)^{T}
$$

The parameter $a \in \mathbb{R}$ corresponds to the on-site (Coulomb) integral and $-t \in \mathbb{R}$ to the hopping (bond) integral (using the standard Hückel vocabulary).

Exploiting the properties of block circulant matrices, one can obtain from $\mathbf{H}_{n}$ the following 
$4 \times 4$ reduced Hamiltonian (note that this actually corresponds to express in reciprocal space the elementary unit cell depicted in Figure 2)

$$
\mathbf{H}(k \theta)=\left[\begin{array}{cccc}
a & -t\left(1+e^{i k \theta}\right) & 0 & 0 \\
-t\left(1+e^{-i k \theta}\right) & a & -t & 0 \\
0 & -t & a & -t\left(1+e^{-i k \theta}\right) \\
0 & 0 & -t\left(1+e^{i k \theta}\right) & a
\end{array}\right]
$$

Notice also, that in $\mathbf{H}(k \theta)$, the dependence on $n$ is now expressed through $\theta=2 \pi / n$.

The reduced Hamiltonian $\mathbf{H}(k \theta)$ commutes with the symmetry operator $\hat{\sigma}_{h}$, therefore its eigenvalues and eigenvectors are most easily found by working in the symmetry-adapted manifolds

$$
\mathcal{L}_{+}=\operatorname{span}\left[\begin{array}{c}
c_{1} \\
c_{2} \\
c_{2} \\
c_{1}
\end{array}\right] \text { and } \mathcal{L}_{-}=\operatorname{span}\left[\begin{array}{c}
c_{1} \\
c_{2} \\
-c_{2} \\
-c_{1}
\end{array}\right]
$$

By projecting $\mathbf{H}(k \theta)$ onto the subspaces spanned by $\mathcal{L}_{+}$and $\mathcal{L}_{-}$, the components $c_{1}, c_{2} \in \mathbb{C}$ can be obtained as the eigenvectors of the $2 \times 2$ effective Hamiltonian

$$
\mathbf{H}^{( \pm)}(k \theta)=\left[\begin{array}{cc}
a & -t\left(1+e^{i k \theta}\right) \\
-t\left(1+e^{-i k \theta}\right) & a \pm t
\end{array}\right]
$$

The eigenvalues of $\mathbf{H}^{(+)}(k \theta)$ are given by

$$
\varepsilon_{ \pm}^{(+)}(k \theta)=a-\frac{t}{2}(1 \pm \sqrt{9+8 \cos k \theta})
$$

while those of $\mathbf{H}^{(-)}(k \theta)$ by

$$
\varepsilon_{ \pm}^{(-)}(k \theta)=a-\frac{t}{2}(-1 \pm \sqrt{9+8 \cos k \theta})
$$

Since $-t<0$, the order of the four eigenvalues, for any $k$, is $\varepsilon_{+}^{(+)}(k \theta)<\varepsilon_{+}^{(-)}(k \theta) \leq \varepsilon_{-}^{(+)}(k \theta)<$ $\varepsilon_{-}^{(-)}(k \theta)$, where we note the alternation between roots of $\mathbf{H}^{(+)}(k \theta)$ and $\mathbf{H}^{(-)}(k \theta)$. Moreover, note that the superscript $( \pm)$ can be viewed as the symmetry label with respect to the $\sigma_{h}$ reflection plane, where $(+)$ stands for symmetric and $(-)$ for antisymmetric.

The eigenvectors of the full Hamiltonian given in Equation (A1) are

$$
|m, k\rangle=\frac{1}{\sqrt{n}} \sum_{\mu=0}^{n-1} e^{\frac{2 \pi i k \mu}{n}}\left[c_{1, m}(k \theta) p_{\mu}^{1}+c_{2, m}(k \theta) p_{\mu}^{2}+c_{3, m}(k \theta) p_{\mu}^{3}+c_{4, m}(k \theta) p_{\mu}^{4}\right]
$$


where $m$ labels the reduced eigenvector of $\mathbf{H}(k \theta)$ given in Equation (A4) and $k$ the quantum number of the rotation $C_{n} .|m, k\rangle$ is eigenvector of the $\hat{C}_{n}$ rotation operator with eigenvalue $e^{\frac{2 \pi i k}{n}}$ and of the reflection operator $\hat{\sigma}_{h}$ with eigenvalue $-(-1)^{m}$.

In Table A1 we report the eigenvalues and the normalized eigenvectors of the reduced Hamiltonian $\mathbf{H}$ given in Equation (A4) expressed as a function of $\xi=k \theta$. Note that for a better readability we have relabeled the eigenvalues according to their energetic order from 1 to 4 as presented in the main text of the manuscript, where 1 is the energetically lowest and 4 the highest.

The expressions of the eigenvectors (and thus the associated eigenvalues) retain the symmetry features of the Hamiltonian. The $1^{\text {st }}$ and $3^{\text {rd }}$ eigenvectors are symmetric by reflection about the plane of the ring, while the $2^{\text {nd }}$ and $4^{\text {th }}$ are antisymmetric as can be inferred from Figure A1.

\section{B Partial Densities of States}

To compute the partial density of states for the energy band $\varepsilon_{1}^{(+)}$, one first inverts the expression as

$$
\begin{aligned}
\varepsilon_{1}^{(+)} & =a-\frac{t}{2}(1+\sqrt{9+8 \cos (\xi)}) \\
\xi & =\arccos \left[\frac{1}{2}\left(\frac{\varepsilon_{1}^{(+)}}{t}-\frac{a}{t}+\frac{1}{2}\right)^{2}-\frac{9}{8}\right]
\end{aligned}
$$

and then computes the derivative with respect to $\varepsilon_{1}^{(+)}$which yields

$$
\begin{gathered}
\frac{\partial \xi}{\partial \varepsilon_{1}^{(+)}}=\frac{\partial}{\partial \varepsilon_{1}^{(+)} \arccos \left[\frac{1}{2}\left(\frac{\varepsilon_{1}^{(+)}}{t}-\frac{a}{t}+\frac{1}{2}\right)^{2}-\frac{9}{8}\right]} \\
=\frac{-\left(\frac{\varepsilon_{1}^{(+)}}{t}-\frac{a}{t}+\frac{1}{2}\right)}{t \sqrt{1-\left[\frac{1}{2}\left(\frac{\varepsilon_{1}^{(+)}}{t}-\frac{a}{t}+\frac{1}{2}\right)^{2}-\frac{9}{8}\right]^{2}}}
\end{gathered}
$$


In a similar way we can derive the partial density of state for $\varepsilon_{2}^{(-)}, \varepsilon_{3}^{(+)}$and $\varepsilon_{4}^{(-)}$, which are given by

$$
\begin{gathered}
\frac{\partial \xi}{\partial \varepsilon_{2}^{(-)}}=\frac{-\left(\frac{\varepsilon_{2}^{(-)}}{t}-\frac{a}{t}-\frac{1}{2}\right)}{t \sqrt{1-\left[\frac{1}{2}\left(\frac{\varepsilon_{2}^{(-)}}{t}-\frac{a}{t}-\frac{1}{2}\right)^{2}-\frac{9}{8}\right]^{2}}} \\
\frac{\partial \xi}{\partial \varepsilon_{3}^{(+)}}=\frac{-\left(\frac{\varepsilon_{3}^{(+)}}{t}-\frac{a}{t}+\frac{1}{2}\right)}{t \sqrt{1-\left[\frac{1}{2}\left(\frac{\varepsilon_{3}^{(+)}}{t}-\frac{a}{t}+\frac{1}{2}\right)^{2}-\frac{9}{8}\right]^{2}}} \\
\frac{\partial \xi}{\partial \varepsilon_{4}^{(-)}}=\frac{-\left(\frac{\varepsilon_{4}^{(-)}}{t}-\frac{a}{t}-\frac{1}{2}\right)}{t \sqrt{1-\left[\frac{1}{2}\left(\frac{\varepsilon_{4}^{(-)}}{t}-\frac{a}{t}-\frac{1}{2}\right)^{2}-\frac{9}{8}\right]^{2}}}
\end{gathered}
$$

\section{Planar Components of the TPS}

The individual integrals contributing to the planar components of the TPS are given in the following expressions

$$
\begin{aligned}
& \langle 2, k|\hat{x}+i \hat{y}| 4, k-1\rangle=\frac{1}{2} \operatorname{Re} \frac{i \pi}{n}\left(-\sqrt{1+\frac{1}{\sqrt{9+8 \cos \left[2(k-1) \pi n^{-1}\right]}}} \sqrt{1-\frac{1}{\sqrt{9+8 \cos \left[2 k \pi n^{-1}\right]}}}\right. \\
& \left.+\frac{16 \cos \left[(k-1) \pi n^{-1}\right] \cos \left[k \pi n^{-1}\right]}{\sqrt{\left.9+8 \cos \left[2(k-1) \pi n^{-1}\right]+\sqrt{9+8 \cos \left[2(k-1) \pi n^{-1}\right.}\right]} \sqrt{9+8 \cos \left[2 k \pi n^{-1}\right]+\sqrt{9+8 \cos \left[2 k \pi n^{-1}\right]}}}\right) \\
& \langle 2, k|\hat{x}-i \hat{y}| 4, k+1\rangle=\frac{1}{2} R e^{-\frac{i \pi}{n}}\left(-\sqrt{1+\frac{1}{\sqrt{9+8 \cos \left[2(k+1) \pi n^{-1}\right]}}} \sqrt{1-\frac{1}{\sqrt{9+8 \cos \left[2 k \pi n^{-1}\right]}}}\right. \\
& \left.+\frac{16 \cos \left[(k+1) \pi n^{-1}\right] \cos \left[k \pi n^{-1}\right]}{\sqrt{\left.9+8 \cos \left[2(k+1) \pi n^{-1}\right]+\sqrt{9+8 \cos \left[2(k+1) \pi n^{-1}\right.}\right]} \sqrt{9+8 \cos \left[2 k \pi n^{-1}\right]-\sqrt{9+8 \cos \left[2 k \pi n^{-1}\right]}}}\right) \\
& \langle 1, k|\hat{x}+i \hat{y}| 3, k-1\rangle=\frac{1}{2} R e^{\frac{i \pi}{n}}\left(-\sqrt{1-\frac{1}{\sqrt{9+8 \cos \left[2(k-1) \pi n^{-1}\right]}}} \sqrt{1+\frac{1}{\sqrt{9+8 \cos \left[2 k \pi n^{-1}\right]}}}\right. \\
& \left.+\frac{16 \cos \left[(k-1) \pi n^{-1}\right] \cos \left[k \pi n^{-1}\right]}{\sqrt{9+8 \cos \left[2(k-1) \pi n^{-1}\right]-\sqrt{9+8 \cos \left[2(k-1) \pi n^{-1}\right]}} \sqrt{9+8 \cos \left[2 k \pi n^{-1}\right]+\sqrt{9+8 \cos \left[2 k \pi n^{-1}\right]}}}\right) \\
& \langle 1, k|\hat{x}-i \hat{y}| 3, k+1\rangle=\frac{1}{2} R e^{-\frac{i \pi}{n}}\left(-\sqrt{1-\frac{1}{\sqrt{9+8 \cos \left[2(k+1) \pi n^{-1}\right]}}} \sqrt{1+\frac{1}{\sqrt{9+8 \cos \left[2 k \pi n^{-1}\right]}}}\right. \\
& \left.+\frac{16 \cos \left[(k+1) \pi n^{-1}\right] \cos \left[k \pi n^{-1}\right]}{\sqrt{9+8 \cos \left[2(k+1) \pi n^{-1}\right]-\sqrt{9+8 \cos \left[2(k+1) \pi n^{-1}\right]}} \sqrt{9+8 \cos \left[2 k \pi n^{-1}\right]+\sqrt{9+8 \cos \left[2 k \pi n^{-1}\right]}}}\right)
\end{aligned}
$$




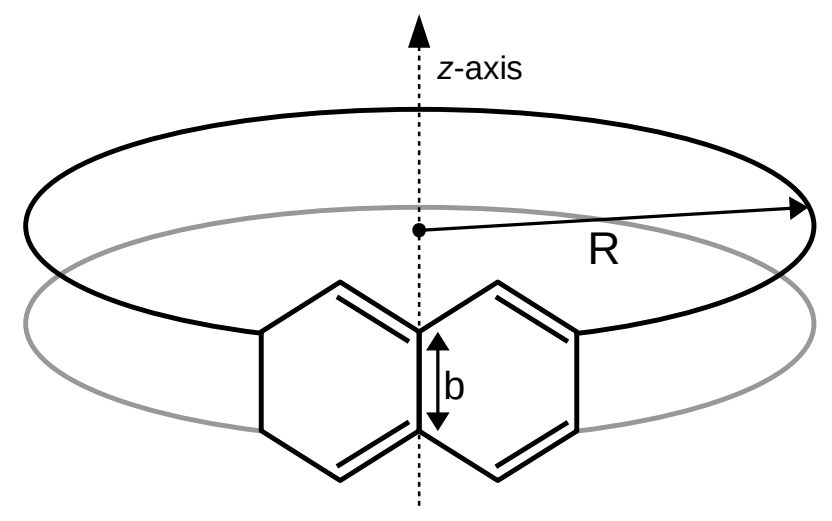

Figure 1: Cyclacene scheme.

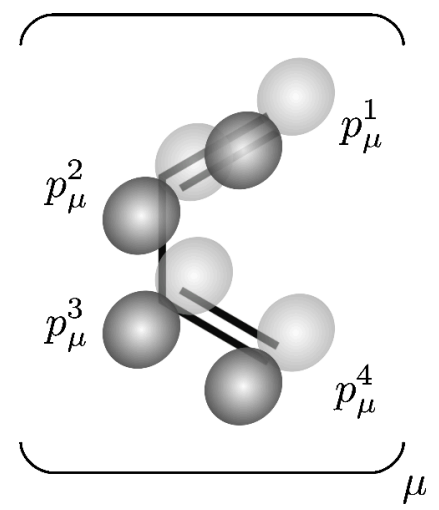

Figure 2: Elementary unit $\mu$ of the polyacene ring with attached basis functions. 


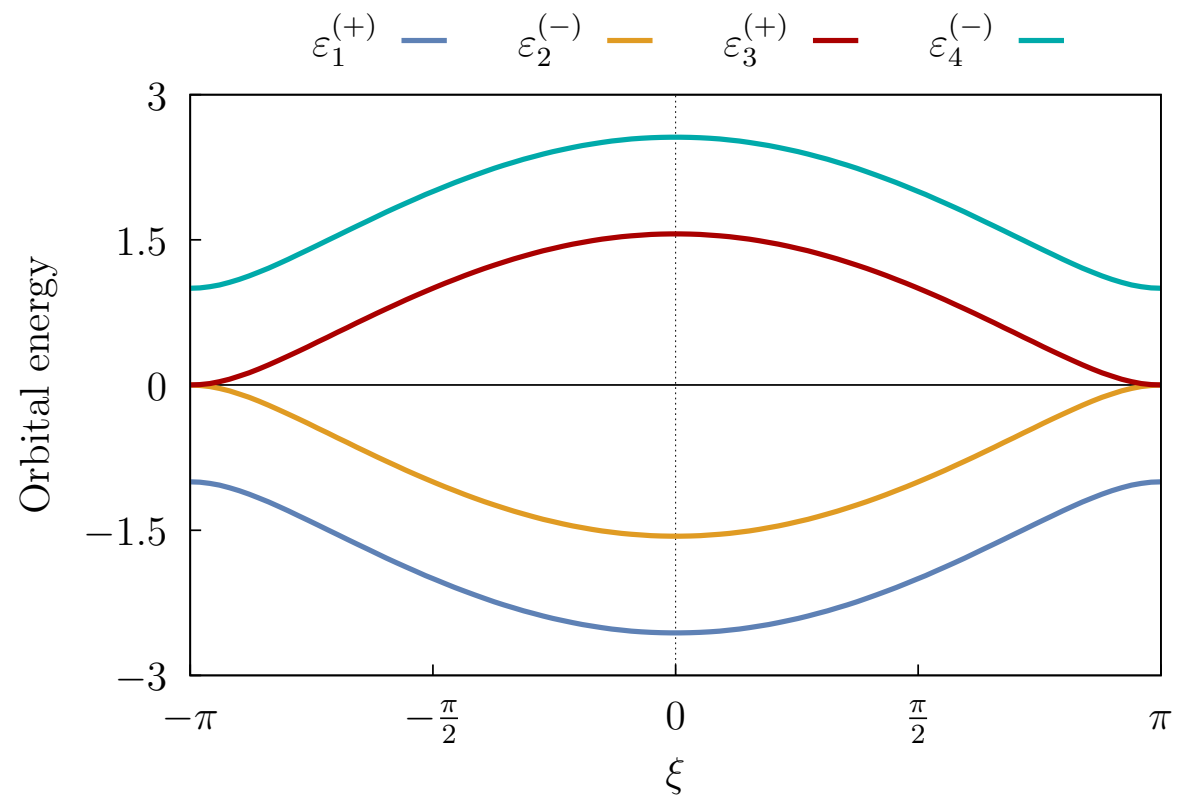

Figure 3: Energy bands (in $t$ units) at Hückel level of theory for $a=0$ and $t=1$.

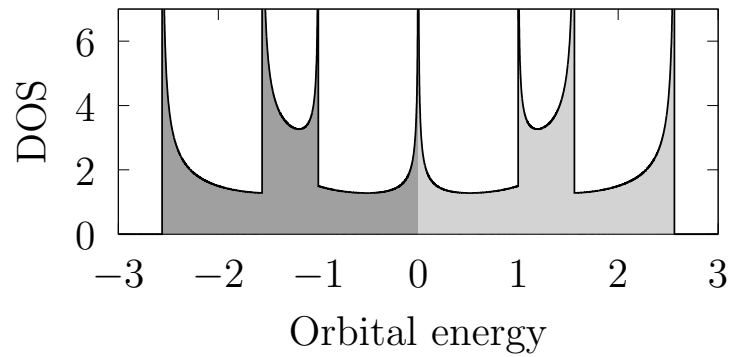

Figure 4: Density of states at Hückel level of theory for $a=0$ and $t=1$. The occupied band is colored in dark gray, while the empty band is in light gray. 

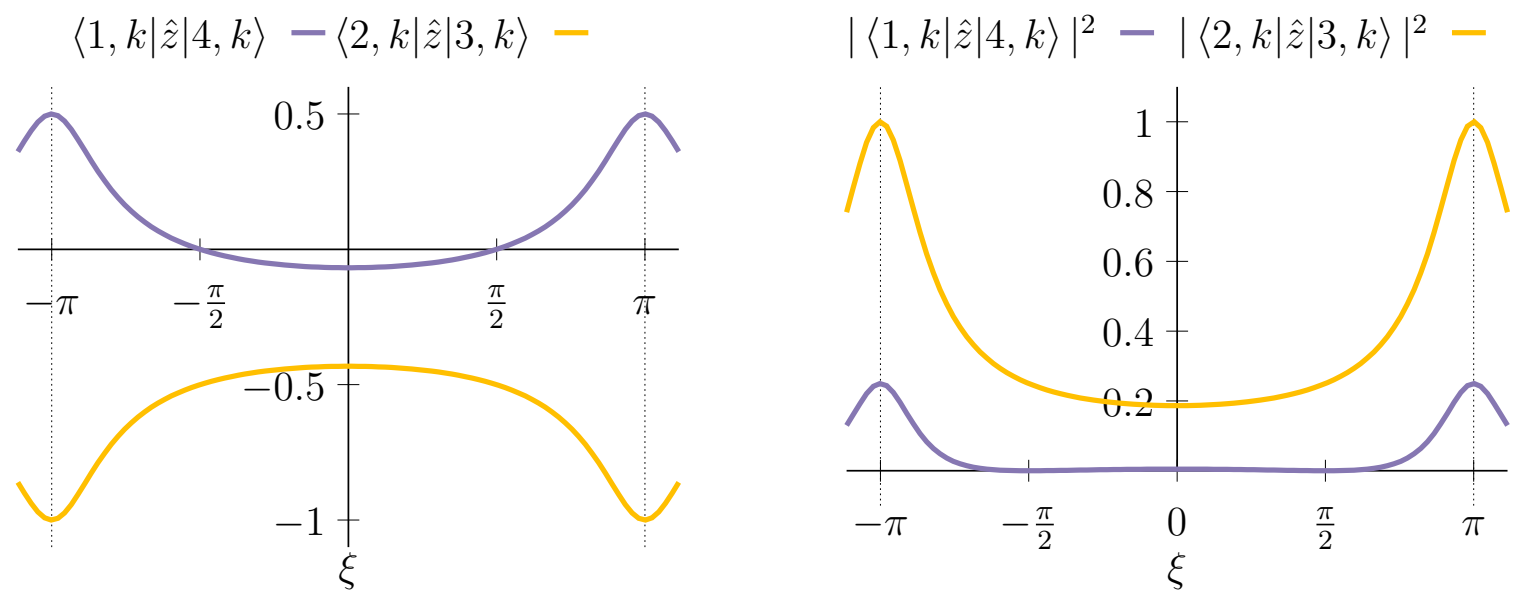

Figure 5: Integral (in $b$ units, i.e. the side length of one hexagon) and squared integral (in $b^{2}$ units) contributions to the axial component of the TPS.
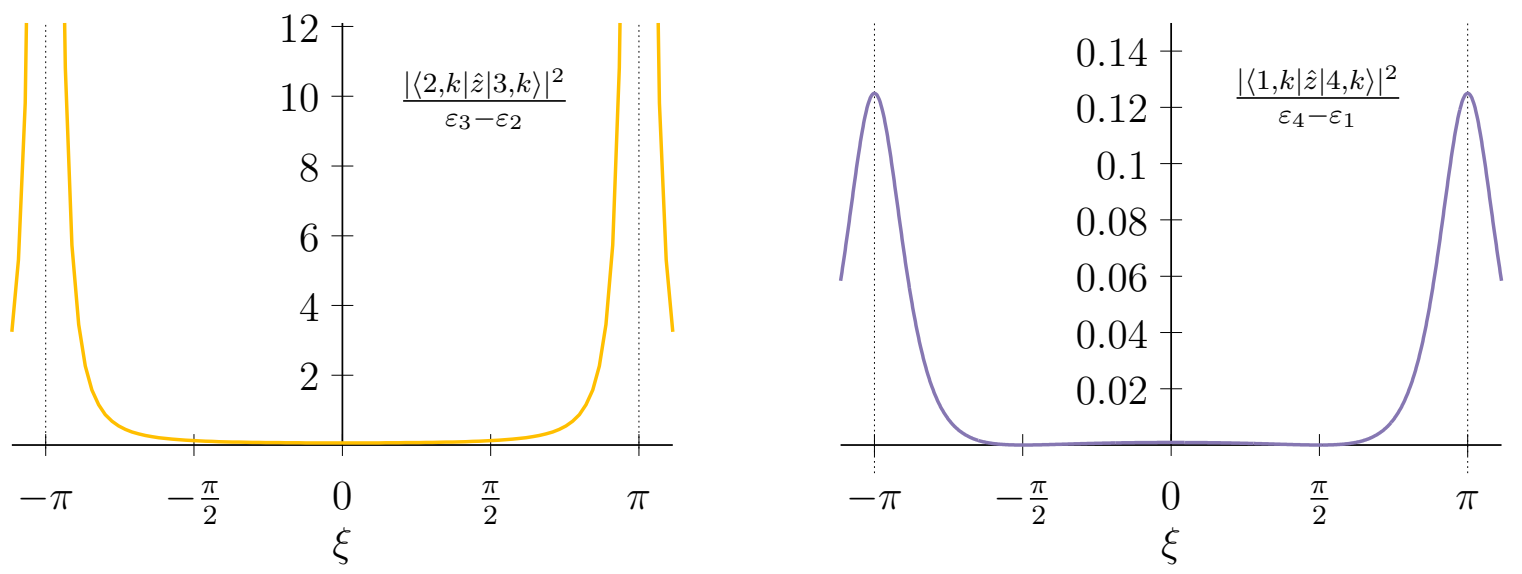

Figure 6: Contributions to the axial component of the polarizability (in $\frac{b^{2}}{t}$ units). Note the different $y$-axis scales. 


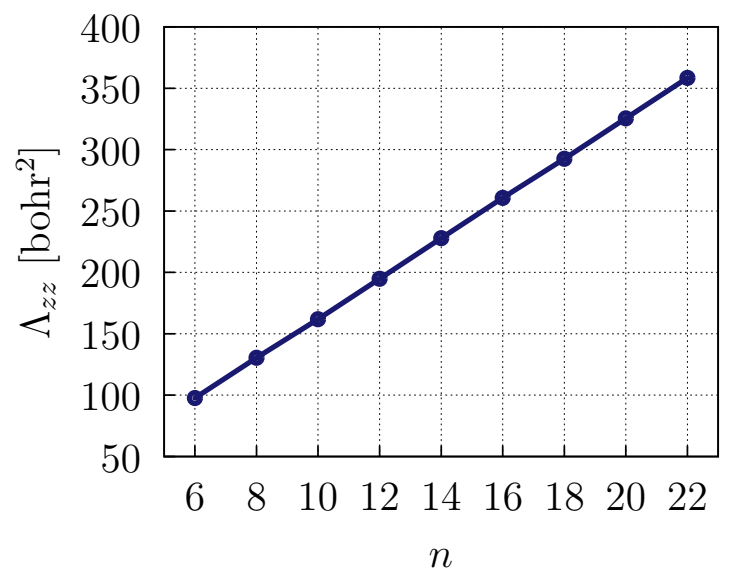

Figure 7: The total axial component of the TPS computed with the CASSCF method.

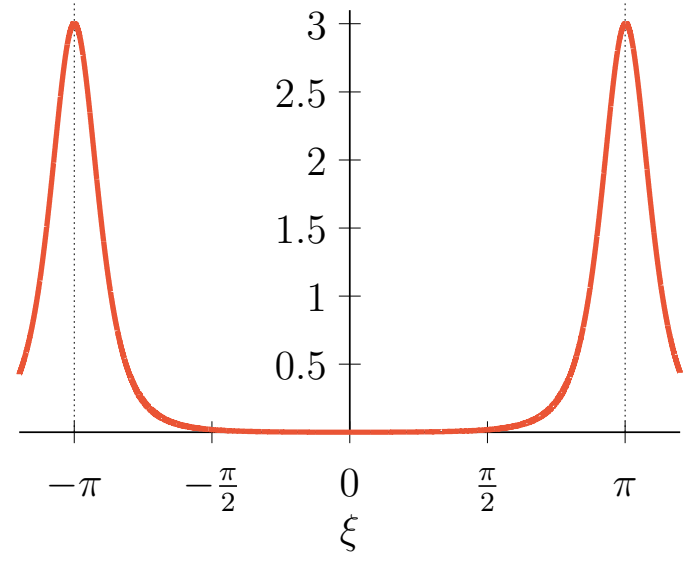

Figure 8: Squared integral contribution (in $b^{2}$ units) to the planar components of the TPS. As usual $b=1$ and $t=1$. 

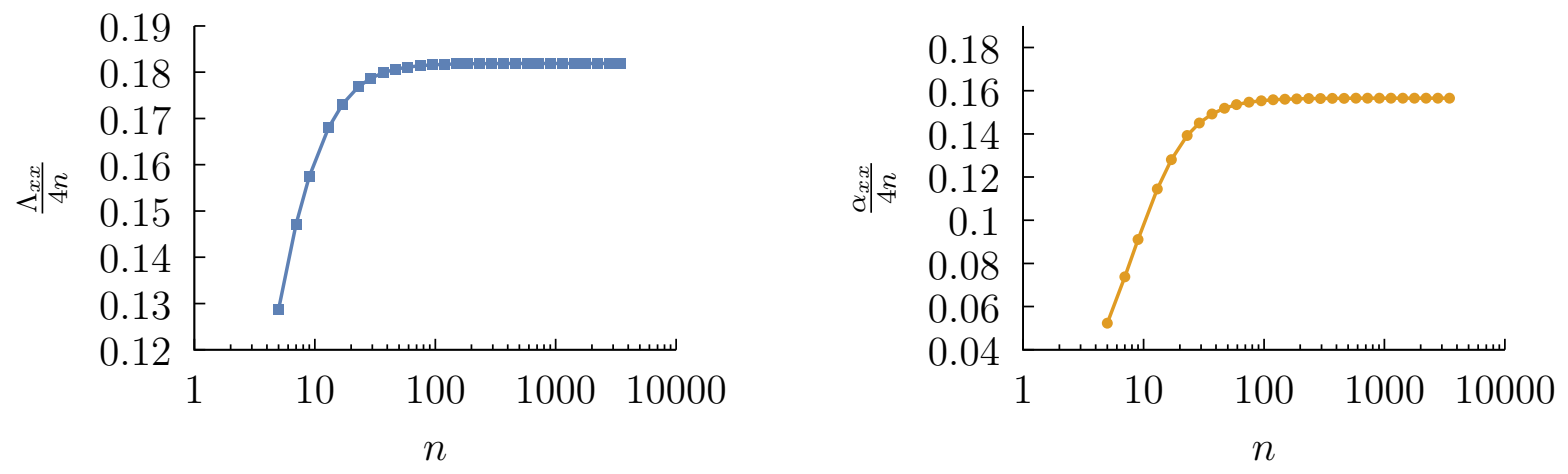

Figure 9: $x$ component of the TPS (left) and polarizability (right) divided by the number of electrons in the system (in $b^{2}$ units and $\frac{b^{2}}{t}$, respectively). 
$|4,0\rangle$
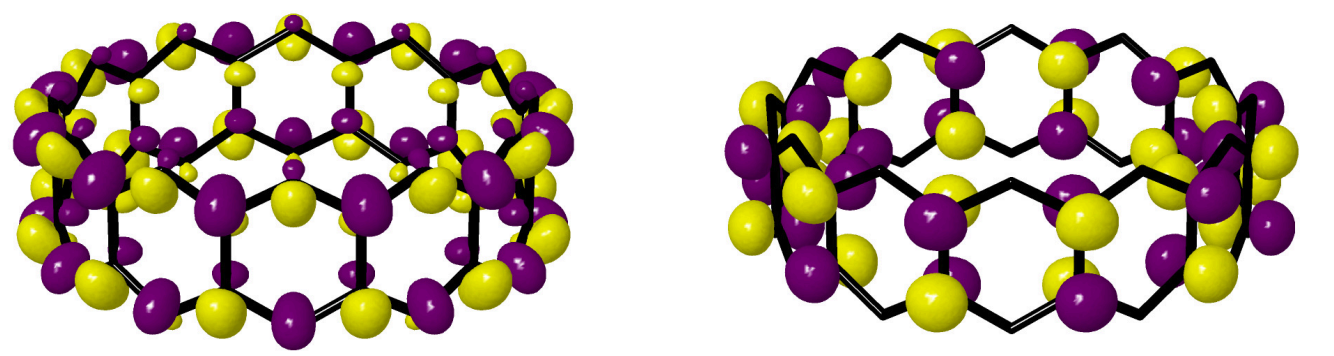

$\left|4, \frac{n}{2}\right\rangle$

$|3,0\rangle$
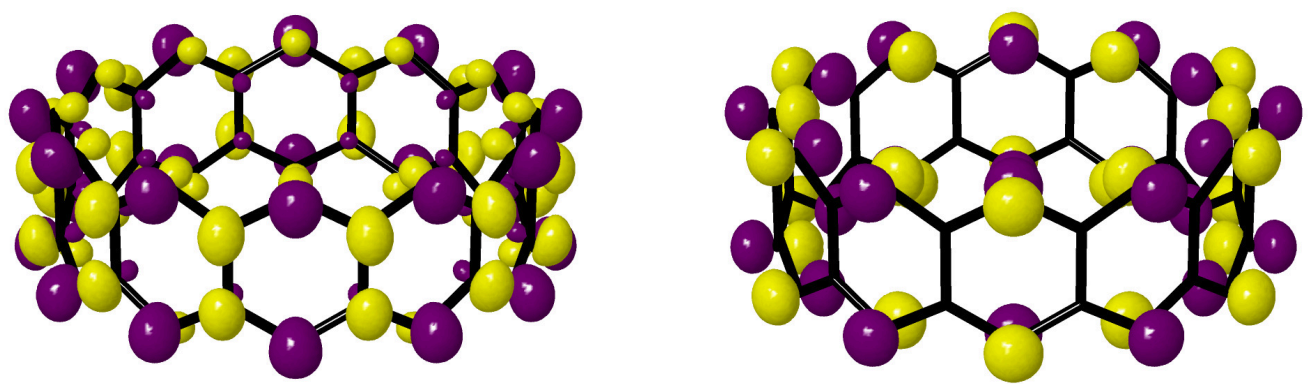

$\left|3, \frac{n}{2}\right\rangle$

$|2,0\rangle$
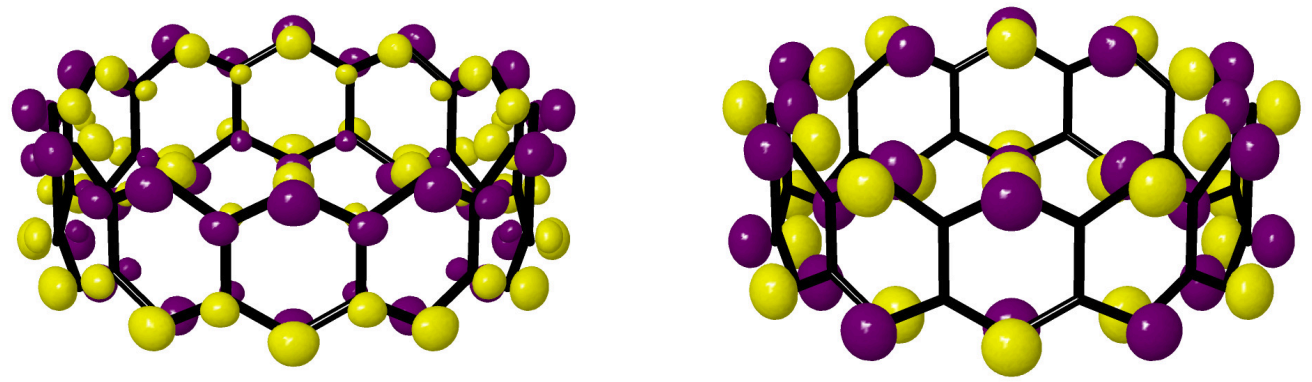

$\left|2, \frac{n}{2}\right\rangle$

$|1,0\rangle$
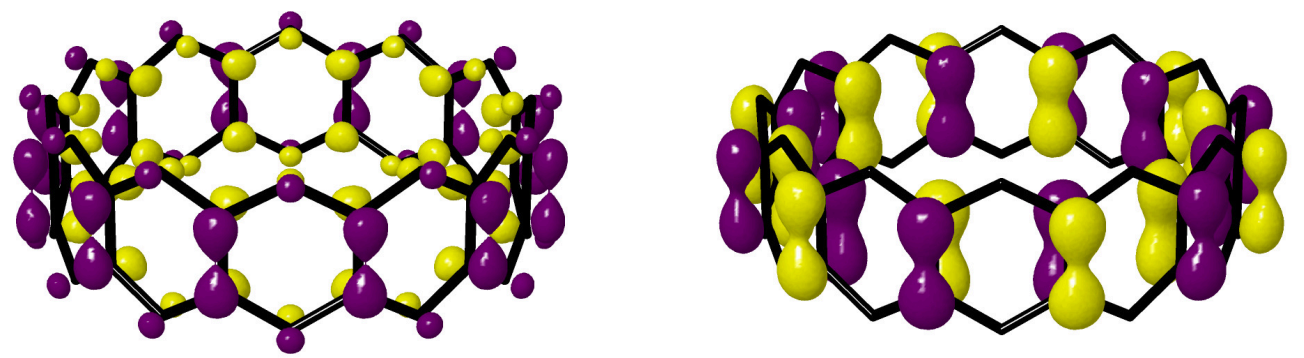

$\left|1, \frac{n}{2}\right\rangle$

Figure A1: Molecular orbitals at $\xi=0$ (left column) and $\xi=\pi$ (right column) for a [12] cyclacene. From bottom to top the energy (eigenvalue) associated to the orbitals increases. Note that the orbitals depicted are canonical Hartree-Fock orbitals using the STO3G basis set, with an isosurface cutoff value of 0.04 . 


\begin{tabular}{|l|ccc|}
\hline atom & $x$ & $y$ & $z$ \\
\hline type 1 & $x_{\mu}^{1}=R \cos \left(\frac{2 \pi(\mu+1 / 2)}{n}\right)$ & $y_{\mu}^{1}=R \sin \left(\frac{2 \pi(\mu+1 / 2)}{n}\right)$ & $z_{\mu}^{1}=b$ \\
type 2 & $x_{\mu}^{2}=R \cos \left(\frac{2 \pi \mu}{n}\right)$ & $y_{\mu}^{2}=R \sin \left(\frac{2 \pi \mu}{n}\right)$ & $z_{\mu}^{2}=\frac{b}{2}$ \\
type 3 & $x_{\mu}^{3}=R \cos \left(\frac{2 \pi \mu}{n}\right)$ & $y_{\mu}^{3}=R \sin \left(\frac{2 \pi \mu}{n}\right)$ & $z_{\mu}^{3}=-\frac{b}{2}$ \\
type 4 & $x_{\mu}^{4}=R \cos \left(\frac{2 \pi(\mu+1 / 2)}{n}\right)$ & $y_{\mu}^{4}=R \sin \left(\frac{2 \pi(\mu+1 / 2)}{n}\right)$ & $z_{\mu}^{4}=-b$ \\
\hline
\end{tabular}

Table 1: Atomic coordinates of the cyclacene. $0 \leq \mu \leq n-1$ labels the elementary unit which is composed by four atoms.

\begin{tabular}{|c|cc|c|}
\hline$n$ & $\alpha_{x x}$ & $\alpha_{z z}$ & NOON H/L \\
\hline 6 & 317.697 & 251.274 & $1.63 / 0.37$ \\
8 & 505.095 & 334.526 & $1.64 / 0.37$ \\
10 & 749.741 & 419.408 & $1.60 / 1.40$ \\
12 & 933.888 & 479.233 & $1.41 / 0.60$ \\
\hline
\end{tabular}

Table 2: Axial and longitudinal components of the polarizability (in atomic units) computed with the CASSCF method. The last column lists the NO occupation numbers of the HOMOLUMO (H/L) pair. 


\begin{tabular}{|c|c|c|c|c|}
\hline & $m=1$ & $m=2$ & $m=3$ & $m=4$ \\
\hline$\varepsilon_{m}^{( \pm)}(\xi)$ & $a-\frac{t}{2}(1+\sqrt{A})$ & $a-\frac{t}{2}(-1+\sqrt{A})$ & $a-\frac{t}{2}(1-\sqrt{A})$ & $a-\frac{t}{2}(-1-\sqrt{A})$ \\
\hline$c_{1, m}(\xi)$ & $+\frac{2 \cos \frac{\xi}{2}}{\sqrt{A+\sqrt{A}}}$ & $-\frac{2 \cos \frac{\xi}{2}}{\sqrt{A-\sqrt{A}}}$ & $+\frac{2 \cos \frac{\xi}{2}}{\sqrt{A-\sqrt{A}}}$ & $-\frac{2 \cos \frac{\xi}{2}}{\sqrt{A+\sqrt{A}}}$ \\
$c_{2, m}(\xi)$ & $+\frac{1}{2} e^{-\frac{i \xi}{2}} \sqrt{1+\frac{1}{\sqrt{A}}}$ & $-\frac{1}{2} e^{-\frac{i \xi}{2}} \sqrt{1-\frac{1}{\sqrt{A}}}$ & $-\frac{1}{2} e^{-\frac{i \xi}{2}} \sqrt{1-\frac{1}{\sqrt{A}}}$ & $+\frac{1}{2} e^{-\frac{i \xi}{2}} \sqrt{1+\frac{1}{\sqrt{A}}}$ \\
$c_{3, m}(\xi)$ & $c_{2, m}(\xi)$ & $-c_{2, m}(\xi)$ & $c_{2, m}(\xi)$ & $-c_{2, m}(\xi)$ \\
$c_{4, m}(\xi)$ & $c_{1, m}(\xi)$ & $-c_{1, m}(\xi)$ & $c_{1, m}(\xi)$ & $-c_{1, m}(\xi)$ \\
\hline
\end{tabular}

Table A1: Eigenvalues and eigenvectors of $\mathbf{H}(\xi)$, with $A=9+8 \cos \xi$. 